\title{
Spatial integration with rats
}

\author{
V. D. CHAMIZO and T. RODRIGO \\ University of Barcelona, Barcelona, Spain \\ and \\ N. J. MACKINTOSH \\ University of Cambridge, Cambridge, England
}

\begin{abstract}
Rats were trained to find the hidden platform in a Morris pool, whose location was defined by reference to a small number of landmarks around the circumference of the pool. In each of three experiments, an experimental group was trained on alternate trials with two different subsets of three of the available landmarks, with the two subsets sharing one landmark in common. When tested with landmarks drawn from both of their training configurations, but without the landmark common to the two sets, they had no difficulty in locating the platform. In Experiment 1, they performed at least as well as a group trained with all the available landmarks present on every trial. In Experiment 2, they performed significantly better than a group trained with two different subsets of landmarks that shared no one landmark in common.
\end{abstract}

When rats explore a novel environment, the chances are that they will be able to perceive only one relatively small part of that environment at a time. How, then, do they locate the route from one part of the environment to another, which have been explored only on separate occasions and include information about the spatial relationships among landmarks that have never been perceived together? One possibility, of course, is that they cannot. The relation among landmarks may be a local affair that, at best, encodes only information about places seen at approximately the same time. Sutherland, Chew, Baker, and Linggard (1987) reported that rats trained to find the hidden platform in a Morris pool were unable to swim directly to the platform from a novel start location unless they had been allowed to swim freely throughout the pool during the course of training. If trained in the platform half of the pool on some trials and allowed to swim in the other half on other trials, they did not swim directly to the platform when released from the latter half.

In the course of exploration, however, animals may learn to combine the location of landmarks into larger configurations that integrate the information in a way that allows them to follow the direct route from one part of the environment to another. Such an idea has been advanced by Worden (1992), who talked of the integration of fragmentary maps into whole maps, by Gallistel (1990), and by Poucet (1993), who talked of "local charts"

This research was supported by grants from the Spanish Ministerio de Educación y Ciencia (SEJ2004-07621-C02) and the Generalitat de Catalunya (AGAUR SGR2005) to V.D.C. Correspondence concerning this article should be addressed either to N. J. Mackintosh, Department of Experimental Psychology, University of Cambridge, Cambridge CB2 3EB, England or to V. D. Chamizo, Universitat de Barcelona, Departament de Psicologia Bàsica, Passeig de la Vall d'Hebron 171, 08035-Barcelona, Spain (e-mail: njsmm@mole.bio.cam.ac.uk, victoria.diez.chamizo@ub.edu). that become linked together. But how does this linking process operate? Poucet suggested that two local charts are combined by the path an animal follows from one to another, which may then allow the animal to specify the direction and distance between the areas represented by the two charts. Alternatively, the link may be established by a common linking place-that is, one that lies on the boundary common to the two areas represented by the initially independent local charts and, therefore, presumably is visible from both.

Although these ideas seem intuitively very plausible, we know of little empirical evidence to substantiate them. The aim of the present series of experiments was to see whether we could obtain such evidence. Rats were trained to swim to the hidden platform in a Morris pool, whose location was defined by a number of landmarks situated around the circumference of the pool. In each experiment reported here, the critical group was one trained on different trials with two separate sets of landmarks, but with one landmark common to the two sets. In order to see whether the presence of this common landmark would allow these animals to integrate the two local charts of the pool established by their training, they were tested for their ability to locate the platform in the presence of one or two landmarks from each training set that did not include this common landmark and which they had, therefore, never seen together before. In Experiment 1, their performance was compared with that of a group trained from the outset with all the landmarks available; in Experiment 2, their performance was compared with that of a group trained on two sets of landmarks that did not share one in common.

\section{GENERAL METHOD}

The apparatus and the general procedures common to all the experiments will be described first. 


\section{Apparatus}

The apparatus was a circular swimming pool, made of plastic and fiber glass, modeled after that used by Morris (1981). It measured $1.58 \mathrm{~m}$ in diameter and $0.65 \mathrm{~m}$ deep and was filled to a depth of $0.49 \mathrm{~m}$ with water rendered opaque by the addition of $1 \mathrm{cl} / \mathrm{L}$ of latex. The water temperature was maintained at $22^{\circ} \pm 1^{\circ} \mathrm{C}$. The pool was situated in the middle of a large room, mounted on a wooden platform $0.43 \mathrm{~m}$ above the floor, and it was surrounded by black curtains reaching from the ceiling to the base of the pool and forming a circular enclosure $2.4 \mathrm{~m}$ in diameter. Inside the black enclosure, around the curtains and hanging from a black false ceiling, a number of objects were placed. They were suspended from the false ceiling, $0.35 \mathrm{~m}$ above the surface of the water and with the midline directly above the wall of the pool. These objects or landmarks defined the location of the platform. In order to ensure that the rats used these landmarks, rather than any inadvertently remaining static room cues, to locate the platform, the landmarks and platform were semirandomly rotated with respect to the room $\left(90^{\circ}, 180^{\circ}, 270^{\circ}\right.$, or $360^{\circ}$ ), with the restriction that all the orientations were used equally each day. A closed circuit video camera with a wide-angle lens was mounted $1.75 \mathrm{~m}$ above the center of the pool inside the false ceiling, and its picture was relayed to recording equipment in an adjacent room. A circular platform, $0.11 \mathrm{~m}$ in diameter and made of transparent Perspex, was mounted on a rod and base and could be placed in one quadrant of the pool, $0.38 \mathrm{~m}$ from the wall, with its top $1 \mathrm{~cm}$ below the surface of the water. The entire false ceiling, with the landmarks suspended, could be rotated from trial to trial, and the platform always rotated with them.

\section{Procedure}

There were three types of trials: pretraining, escape training, and test trials. Pretraining consisted of placing a rat into the pool, facing the wall, without landmarks but with the platform present. The rat was given $180 \mathrm{sec}$ to find the platform and, once it had found it, was allowed to stay on it for $30 \mathrm{sec}$. If it had not found the platform within the $180 \mathrm{sec}$, it was picked up, placed on it, and left there for $30 \mathrm{sec}$. The platform was moved from one trial to the next, and the rat was placed in the pool in a different location on each trial, as far as possible equally often on the same or the opposite side of the pool from the platform and with the platform to the right or to the left of where the rat was placed. The four start positions used throughout each experiment were at A, C, E, and $\mathrm{C}^{\prime}$ in Figure 1. The rats in all the experiments were always given five such pretraining trials spread over 3 days, one trial on Day 1 and two trials on each of Days 2 and 3.

The procedure for escape training was exactly the same as that for pretraining, except that a configuration of several landmarks was

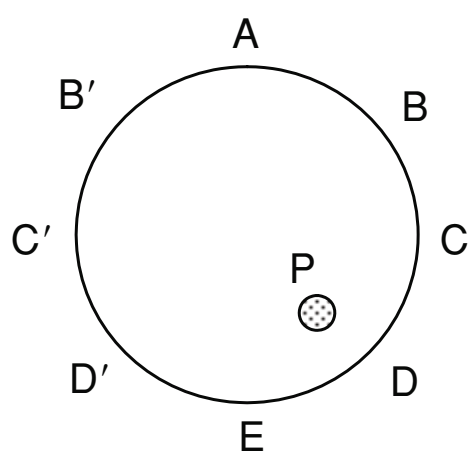

Figure 1. A schematic representation of the pool and the five landmarks used in Experiment 1 (A, B, C, D, and E) and in Experiments 2 and $3\left(A, B^{\prime}, C^{\prime}, D^{\prime}, E\right.$, and $\left.D\right)$, as well as the platform (P). (In Experiment 3, there were five landmarks only: $A$, $B^{\prime}, C^{\prime}, D^{\prime}$, and E.) always present and the animals were given only $120 \mathrm{sec}$ to find the platform. There were always eight escape trials per day, with an average intertrial interval of 10-15 $\mathrm{min}$, and the platform and the landmarks were rotated between trials, with the platform always placed at a fixed position in relation to the landmarks in each experiment.

After the training phase, all the rats received several test trials. A test day consisted of eight retraining escape trials, followed by a single test trial. Escape trials were the same as those in training for each group. A test trial consisted of placing the rat into the pool, with landmarks present but without the platform, and leaving it there for $60 \mathrm{sec}$ (except in Experiment 1, in which the test trial lasted $120 \mathrm{sec}$ ). The four starting positions were the same as those used in training. For purposes of recording the rats' behavior, on test trials, the pool was divided into four quadrants. In Figure 1, the quadrants were A-C, C-E (the platform quadrant), $\mathrm{E}-\mathrm{C}^{\prime}$, and $\mathrm{C}^{\prime}-\mathrm{A}$. The amount of time the rats spent in the platform quadrant was recorded. A significance level of $p<.05$ was adopted for the statistical tests reported in all the experiments.

\section{EXPERIMENT 1}

\section{Method}

Subjects and Apparatus. The subjects were 26 Long-Evans rats, 13 males and 13 females, approximately 5 months old at the beginning of the experiment; they had previously participated in a taste aversion experiment. They were divided into two groups: Group Int (for integration), with 6 males and 7 females, and Group Config (for configuration), with 7 males and 6 females, matched for latency to find the platform on the pretraining trials. The rats were caged in groups of 2, and they were maintained on ad lib food and water, in a colony room which had a 12:12-h light:dark cycle. They were tested within the first $8 \mathrm{~h}$ of the light cycle.

There were five landmarks used in this experiment, located at positions A to E in Figure 1. They were as follows: at A, a 40-W fixed light placed inside a white plastic inverted cone $11 \mathrm{~cm}$ high and $13 \mathrm{~cm}$ in diameter at the base; at B, a white cardboard cube (20-cm edge) with a black circle at the center of each side (9.5-cm diameter); at C, a 15-cmdiameter plastic beach ball with mixed colors; at $\mathrm{D}$, a white cardboard cone $16 \mathrm{~cm}$ in diameter and $59 \mathrm{~cm}$ in height, with 1-cm-thick black horizontal stripes spaced $3.5 \mathrm{~cm}$ apart; and at $\mathrm{E}$, an intermittent $1-\mathrm{W}$ light flashing on and off at a frequency of 60-80 times per minute. In the description of the experimental procedure that follows, these five landmarks will be referred to by the letters A, B, C, and so forth. The platform was located in front of $\mathrm{D}$, as shown in Figure 1.

Procedure. After pretraining, Group Int received 10 days of escape training, for a total of 80 trials, with three landmarks present on each trial. On a random half of the trials each day, the landmarks were $\mathrm{A}, \mathrm{B}$, and $\mathrm{C}$, and for the remaining trials, $\mathrm{C}, \mathrm{D}$, and $\mathrm{E}$ (with $\mathrm{C}$ common to each configuration). Group Config was trained with all five landmarks present on all trials each day. To equate their experience with all the landmarks save C, the animals in Group Config were trained only every other day, for a total of 40 trials.

At the end of training, all the rats received 3 test days. For Group Int, each day consisted of eight retraining trials, followed by a single test; to preserve Group Config's equal experience with the landmarks, they received only four retraining trials before the test trial each day. On Day 1, the animals were tested in the presence of the training configurations (i.e., TC) for Group Int - half the animals in each group with $\mathrm{A}, \mathrm{B}$, and $\mathrm{C}$, and the remainder with $\mathrm{C}, \mathrm{D}$, and $\mathrm{E}$. On Day 2, the animals were tested in the presence of three landmarks, without landmark $\mathrm{C}$, the landmark common to the two training configurations for Group Int (i.e., test InNC); half of each group was tested with A, E, and B, and half with A, E, and D. On Day 3, all the animals were tested with $\mathrm{A}$ and $\mathrm{E}$ (i.e., the two-cue test).

\section{Results and Discussion}

The animals' escape latencies decreased over the training phase from a mean of $34.18 \mathrm{sec}$ on Day 1 to a mean 
of $9.22 \mathrm{sec}$ on Day 10 for Group Int and from a mean of $24.18 \mathrm{sec}$ on Day 1 to $9.87 \mathrm{sec}$ on their last day of training for Group Config; an ANOVA on this set of scores revealed a significant effect of days $[F(1,24)=83.21, p<$ $.001]$ and a significant interaction between groups and days $[F(1,24)=6.12, p<.05]$. Analysis of simple effects revealed, however, that the two groups did not differ on their last day of training $(F<1)$. There was equally no overall difference between the two groups' overall latencies on the retraining trials that preceded the three test trials $(F<1)$.

The results of the test trials are shown in Figure 2. Scores marked with an asterisk are significantly above the chance level of $30 \mathrm{sec}$ spent in the platform quadrant (minimum $t=4.1$ ). As can be seen, both groups performed equally well on Tests 1 and 2, but when tested with only two landmarks, one from each training configuration, Group Int performed more accurately than Group Config, although neither group was significantly above chance [for Group Int, $t(12)=1.90, p<.10$ ]. An ANOVA with factors of groups and tests revealed a significant effect of tests $[F(2,48)=30.46, p<.001]$ and a significant interaction between groups and tests $[F(2,48)=3.79, p<.05]$. A simple effects analysis of this interaction revealed that the groups differed on Test 3 , with two landmarks $[F(2,48)=$ $5.33, p<.01]$, but not on the other two tests $(F<1)$.

It is clear that Group Int performed at least as well as Group Config on tests with either three or two landmarks drawn from the two configurations on which they were trained, even when the landmark common to the two configurations was not present. This would seem to suggest that they had no difficulty integrating the information provided about the location of the platform from their two types of training trials. For both groups, of course, there was a difference between the landmarks present on Tests 2 and 3 and those present on their training trials. This might be expected to have resulted in some generalization decrement. Is it possible to explain the pattern of results observed and, in particular, the superior performance of Group Int on the two-cue test by appealing to generalization decrement? It might seem that the change from five landmarks to two, for Group Config, was greater than the change from three to two, for Group Int. But at least one formal account of generalization predicts the opposite. According to Pearce (1994), generalization between two compound stimuli is determined by the product of the proportion of the component stimuli in each compound that are shared with the other compound. In the case of Group Config, the test configuration of A and E shares two of the five component stimuli present in their training configuration, so generalization from training to test will be .40 . In the case of Group Int, the test configuration of $A$ and $E$ shares one of its two components (A) with training configuration $\mathrm{A}, \mathrm{B}$, and $\mathrm{C}$ and its other component $(\mathrm{E})$ with the other training configuration, C, D, and E. So half of the component stimuli of $\mathrm{A}$ and $\mathrm{E}$ is the same as one of the three components of one training compound, and the other half is the same as one of the three components of the other training compound. Pearce's formula states that this will result in a total generalization of .33 (being one half times one third from each training configuration) - that is, less than the generalization seen in Group Config.

The question remains whether the good test performance shown by Group Int was, indeed, dependent on the fact that they were trained with one landmark shared in common by their two training configurations. Experiment 2 tested this.

\section{EXPERIMENT 2}

\section{Method}

Subjects and Apparatus. The subjects were 16 naive Hooded Lister rats, 8 males and 8 females approximately 4 months old at

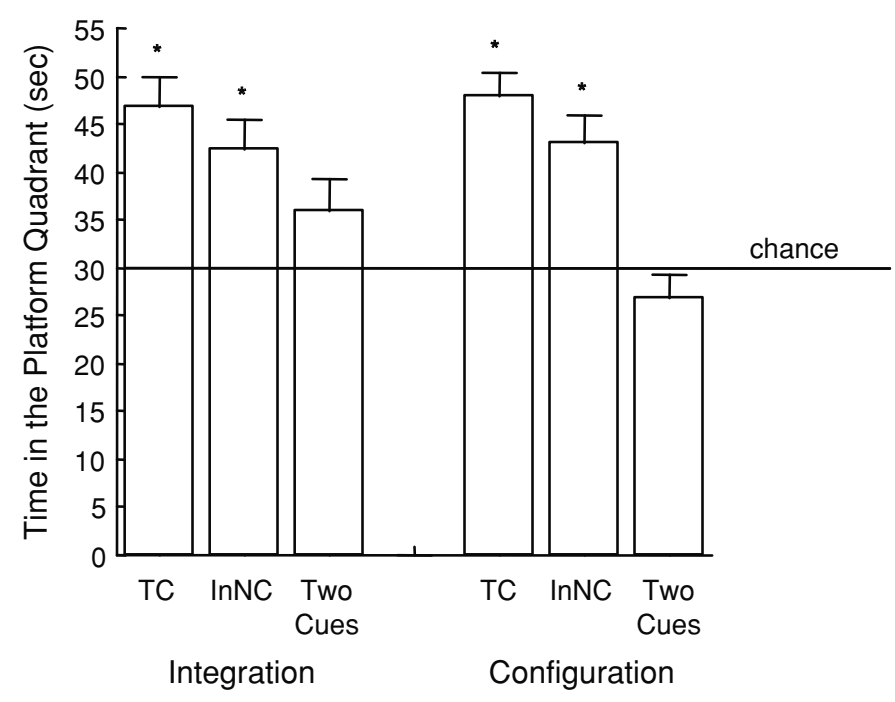

Figure 2. Mean time spent in the platform quadrant for the two groups, Group Integration and Group Configuration, in Experiment 1 during the three tests (TC, InNC, and Two Cues). 
the beginning of the experiment; they had previously participated in a taste aversion experiment. They were divided into two groups, Group Int and Group Con, matched for sex and for latency to find the platform on pretraining trials. The animals were housed and maintained as in Experiment 1, and the apparatus was the same as before, except for some change in the landmarks used and in their position. The landmarks were located at positions $A, B^{\prime}, C^{\prime}, D^{\prime}, E$, and $\mathrm{D}$ as follows: at A, the previous fixed light; at $\mathrm{B}^{\prime}$, three mop heads attached together forming a cylindrical figure $12 \mathrm{~cm}$ in diameter and $22 \mathrm{~cm}$ high; at $C^{\prime}$, a green plastic plant approximately $35 \mathrm{~cm}$ in diameter and $30 \mathrm{~cm}$ high; at $\mathrm{D}^{\prime}$, the previous inverted flowerpots; at $\mathrm{E}$, the previous intermittent light; and at $\mathrm{D}$, the previous cone.

Procedure. After pretraining exactly as in Experiment 1, in the training phase, all the rats received eight trials a day for 12 days. There were three landmarks available on each trial, as shown in Figure 1. For Group Int, on half the trials each day, these landmarks were A, B', and $\mathrm{C}^{\prime}$, and on the remainder, they were $\mathrm{C}^{\prime}, \mathrm{D}^{\prime}$, and $\mathrm{E}$ (where $\mathrm{C}^{\prime}$ was the landmark common to the two configurations), and for Group Con they were $\mathrm{A}, \mathrm{B}^{\prime}$, and $\mathrm{C}^{\prime}$ on half the trials and $\mathrm{D}^{\prime}, \mathrm{E}$, and $\mathrm{D}$ on the remainder (no landmark was common to the two configurations). Other details of training were the same as those in Experiment 1.

After the training phase, all the rats received 8 test days; as in Experiment 1 , each day consisted of eight retraining trials, followed by a single test trial on which the platform was removed from the pool and the rats were allowed to swim in the pool for $60 \mathrm{sec}$. There were four different types of test trial (TC, InC, InNC, and InNC2), each of them presented twice. Test TC was in the presence of one of the training configurations. Half the animals in each group were tested with one configuration on both days, and the remainder with the second configuration (either $\mathrm{A}, \mathrm{B}^{\prime}$, and $\mathrm{C}^{\prime}$ or $\mathrm{C}^{\prime}, \mathrm{D}^{\prime}$, and $\mathrm{E}$ for Group Int, and either $\mathrm{A}, \mathrm{B}^{\prime}$, and $\mathrm{C}^{\prime}$ or $\mathrm{D}^{\prime}, \mathrm{E}$, and $\mathrm{D}$ for Group Con). This test was conducted on Days 1 and 8 of the test phase. The second and the third tests were both integration tests, with and without the common landmark present (Test InC and Test InNC, respectively). Test $\mathrm{InC}$ was in the presence of $\mathrm{A}, \mathrm{C}^{\prime}$, and $\mathrm{E}$ for all the rats (with the same landmarks on both days), and Test InNC with Landmarks A, $\mathrm{B}^{\prime}$, and $\mathrm{E}$ (for half the animals in each group on both days) or with $\mathrm{A}, \mathrm{D}^{\prime}$, and $\mathrm{E}$ (for the remainder on both days). Test InC was given on Days 2 and 5, and Test InNC on Days 3 and 4. The final test, InNC2, occurred on Days 6 and 7. This test was similar to Test InNC, but with only two landmarks, one from each training configuration, A and $\mathrm{D}^{\prime}, \mathrm{A}$ and $\mathrm{E}, \mathrm{B}^{\prime}$ and $\mathrm{D}^{\prime}$, or $\mathrm{B}^{\prime}$ and $\mathrm{E}$. Half the rats in each group were tested on $\mathrm{A}$ and $\mathrm{D}^{\prime}$ on one day and $\mathrm{A}$ and $\mathrm{E}$ on the other (order counterbalanced), and the remainder on $\mathrm{B}^{\prime}$ and $\mathrm{D}^{\prime}$ on one day and $\mathrm{B}^{\prime}$ and $\mathrm{E}$ on the other.

\section{Results and Discussion}

The animals' escape latencies decreased over training from a mean of $32.49 \mathrm{sec}$ on Day 1 to a mean of $11.01 \mathrm{sec}$ on Day 12 for Group Int and from 49.08 to $11.76 \mathrm{sec}$ for Group Con; there was a significant effect of days $[F(11,154)=$ $22.64]$, and the interaction with group was also significant $[F(11,154)=2.34]$. Additional analyses of the interaction between groups and days showed that the two groups differed on Day 1 only $(p<.05)$. On the retraining trials that preceded the eight test trials, latencies had reached asymptote, and there was no further change $(F \mathrm{~s}<1.5)$.

Figure 3 shows the time spent in the platform quadrant by the two groups over all test trials. Scores marked with an asterisk differed significantly from chance by paired means $t$ tests (minimum $t=3.17$ ). As can be seen, Group Int performed above chance on all four tests, whereas Group Con was significantly above chance only on the TC and InC tests. An ANOVA taking into account the groups and tests variables showed a significant effect of tests $[F(3,42)=4.01]$; the interaction of groups and tests was also significant $[F(3,42)=4.79]$, as well as the triple interaction of groups, tests, and days $[F(3,42)=6.12]$. A simple effects analysis of the interaction between groups and tests showed that the two groups did not differ on Test TC, Test InC, or Test InNC $\left(F_{\mathrm{s}}<2\right)$ but clearly differed on Test InNC2 $[F(1,14)=12.51]$. An analysis of the triple groups $\times$ tests $\times$ days interaction showed that the interaction between groups and tests was significant on both days $[$ Day $1, F(3,42)=4.10$; Day $2, F(3,42)=6.92]$. A simple effects analysis of these interactions showed that on Day 1, the two groups differed only on Test TC $[F(1,14)=6.39]$ and on Test InNC2 $[F(1,14)=11.43]$. On both tests, the performance of Group Int was better than that of Group

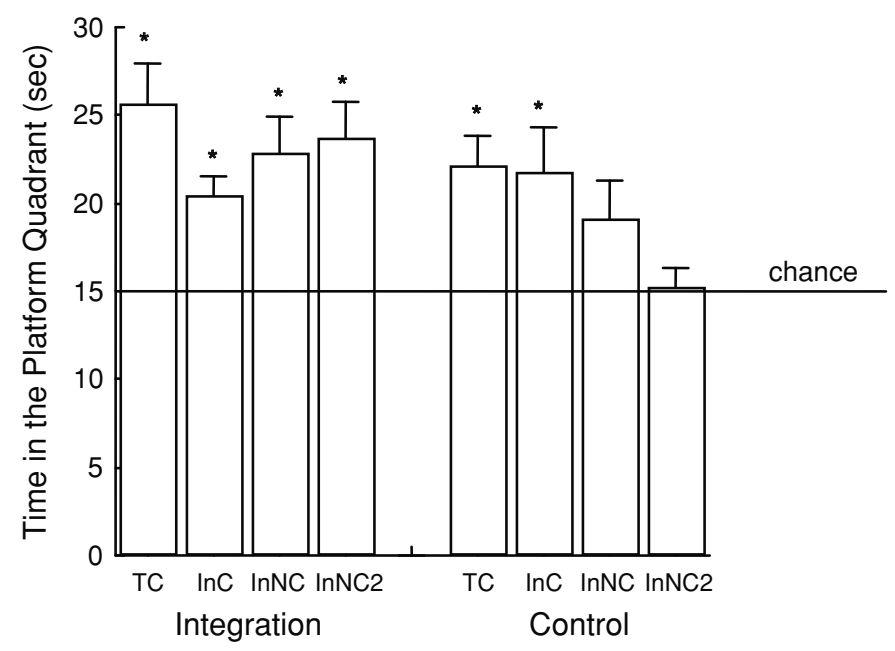

Figure 3. Mean time spent in the platform quadrant for the two groups, Group Integration and Group Control, in Experiment 2 during the four tests (TC, InC, InNC, and InNC2). 
Con. On Day 2, the two groups differed only on Tests $\operatorname{InNC}[F(1,14)=9.55]$ and $\operatorname{InNC} 2[F(1,14)=6.49]$. On both tests, the performance of Group Int was again better than that of Group Con.

The results of this experiment are reasonably clear. Group Int, trained on two different configurations of landmarks that shared one landmark in common, performed significantly above chance on test trials to subsets of these landmarks that did not contain the common landmark (Tests InNC and InNC2), whereas Group Con, trained on two nonoverlapping sets of landmarks, did not. Although the difference between the two groups on Test InNC was significant only on Day 2, on Test InNC2 there was a significant difference between the two groups on both days. Perhaps equally remarkably, the two groups did not differ on Test InC (i.e., when tested with a set of three landmarks when this set did contain the common landmark), where one might have expected that the extra training received by Group Int with this landmark would have benefited their performance.

Group Con performed significantly above chance on Test InC, and although not above chance on Test InNC, their performance here was not greatly inferior to that of Group Int. This might be taken as evidence of at least some integration of information from their two training configurations, even though the configurations contained no common landmark. A more reasonable interpretation is to note that both of these tests always contained two landmarks from one training configuration, and the performance of Group Int on Test InNC2 shows that two landmarks are sufficient to locate the platform.

It is worth commenting on one possible explanation of the poor performance of Group Con on Test InNC2. Unlike Group Int, their initial training trials had included trials with Landmark D, which was closer to the platform than any other. It is conceivable that the presence of D overshadowed learning about Landmarks E and D', which were present on the same trials. Chamizo, Manteiga, Rodrigo, and Mackintosh (2006; for a related study, see also Spetch, 1995) have shown that a landmark very close to the platform is able to overshadow ones farther away. If so, Group Con might have been expected to perform poorly whenever tested with Landmarks E or D' (one or the other of which was present on all of Tests InNC2). There are several reasons to discount this possibility. First, it would equally predict that Group Con should have performed poorly on Test InC, which they did not. Second, if Landmark D was so effective, one might expect that, on Test TC, those animals in Group Con tested with E, D', and $\mathrm{D}$ would have performed more accurately than those tested with $\mathrm{A}, \mathrm{B}^{\prime}$, and $\mathrm{C}^{\prime}$. On this first test trial, there was no difference between the two subgroups: Those tested with $\mathrm{E}, \mathrm{D}^{\prime}$, and D spent $21.09 \mathrm{sec}$ in the platform quadrant, whereas those tested with A, B', and $C^{\prime}$ spent $20.69 \mathrm{sec}$ in the platform quadrant. Third, if $\mathrm{E}$ and $\mathrm{D}^{\prime}$ were overshadowed and the animals used the two landmarks from one training configuration (rather than integrating all three landmarks) to find the platform on Test InNC, the animals tested with A, E, and $\mathrm{D}^{\prime}$ should have performed worse than those tested with A, B', and E. There was no such difference: Those tested with $\mathrm{A}, \mathrm{E}$, and $\mathrm{D}^{\prime}$ spent $18.95 \mathrm{sec}$ in the platform quadrant, whereas those tested with A, B', and $E$ spent $19.31 \mathrm{sec}$ in the platform quadrant. Finally, it should be noted that the overshadowing effect observed by Chamizo et al. relied on a more extreme difference in spatial proximity to the platform than was involved here: They found that the control maintained by a landmark a moderate distance away from the platform was less when it was trained in conjunction with one next to the platform than when it was trained with one on the opposite side of the pool. It is perhaps also worth noting that there is just as much reason to expect that in Group Int, Landmark $\mathrm{C}^{\prime}$, which was trained for twice as many trials as any other landmark, should have overshadowed all the landmarks shown in Tests InNC and InNC2.

\section{EXPERIMENT 3}

The performance of Group Int on Test InNC2 in Experiment 2, with two landmarks which they had never seen together before, seems good evidence of integration. It is possible, however, that these animals were not relying on the configuration of the two landmarks to locate the platform, but simply on a single landmark, A, B', D', or E alone. Experiment 3 was designed to test this possibility by including a test with a single landmark. Of course, it is worth noting that if it were possible to locate the platform by using a single landmark, this should have been equally possible for Group Con - in which case, they would presumably have performed above chance on the two-cue test.

\section{Method}

Subjects and Apparatus. The subjects were 6 naive Hooded Lister males, approximately 5 months old at the beginning of the experiment, that had previously participated in a taste aversion experiment. They were kept and maintained as in the previous experiments. The apparatus and landmarks were the same as those in Experiment 2, except that since only Group Int was run in this experiment, only five landmarks $\left(\mathrm{A}, \mathrm{B}^{\prime}, \mathrm{C}^{\prime}, \mathrm{D}^{\prime}\right.$, and $\left.\mathrm{E}\right)$ were used.

Procedure. The general procedure was exactly the same as that in Experiment 2, with only one exception. Each animal received only three different types of test (TC, InNC2, and one-cue [1Cue]).

All 6 animals were trained in exactly the same way as Group Int in Experiment 2, except that training continued for 14 days, rather than 12. After the training phase, all the rats were tested for 6 days. Test TC occurred on Days 1 and 6, and Test InNC2 on Days 2 and 3. The procedure for these tests was exactly the same as that in Experiment 2. On Days 4 and 5, the rats were tested with a single landmark, 3 rats with $\mathrm{A}$ on one day and $\mathrm{E}$ on the other, and 3 with $\mathrm{B}^{\prime}$ on one day and $\mathrm{D}^{\prime}$ on the other.

\section{Results and Discussion}

The animals' escape latencies decreased over the course of training from a mean of $39.99 \mathrm{sec}$ on Day 1 to one of $9.49 \mathrm{sec}$ on Day 14; there was a significant effect of days $[F(13,65)=16.03]$. On this occasion, there was a further decrease in latency from 10.16 to $6.31 \mathrm{sec}$ over the course of the retraining trials that preceded the test trials $[F(5,25)=7.31]$. 
Figure 4 shows the time spent in the platform quadrant by the rats over all the test trials. Scores marked with an asterisk differed significantly from chance by paired means $t$ tests (minimum $t=3.21$ ). An ANOVA taking into account the variables of tests and days showed that the variable tests was significant $[F(2,10)=7.54]$. No other main effect or interaction was significant $(F \mathrm{~s}<1)$. A Newman-Keuls test showed that $(\mathrm{TC}=$ InNC2 $) \neq$ 1Cue. As was the case with Group Int in Experiment 2, the animals performed as well with two landmarks, one from each training configuration, as they did with their original training configurations, but it is clear that they were unable to locate the platform when tested with only a single cue.

\section{GENERAL DISCUSSION}

The results of these three experiments provide good evidence that if rats are trained in a Morris pool with two different configurations of landmarks signaling the location of the hidden platform on different trials, the presence of one landmark common to the two configurations allows them to integrate what they learn about the location of the platform from the two types of training trials. Evidence of such integration was found by giving test trials with a subset of landmarks, some from one training configuration, some from the other, but without the one landmark common to both configurations. In Experiment 1, the animals that had received such training performed at least as well on such test trials as those trained from the outset with all five landmarks from both configurations present on all trials. Indeed, when tested with only two landmarks, one from each configuration, they performed significantly more accurately. Experiments 2 and 3 confirmed that rats trained in this way could perform significantly above chance when tested with one landmark from each configuration. Experiment 3, however, also showed that they are unable to locate the platform when tested with only a single landmark. Thus, the results of the three experiments may be taken to mean that they can locate the platform by using a novel configuration of two landmarks that they have never seen together before-provided that they have been trained on two configurations containing not only these landmarks, but also one landmark common to each.

Experiment 2 was designed to test the proposition that such integration did, indeed, depend on the presence of one landmark common to the two training configurations. In this experiment, Group Int was trained with, and Group Con without, such a common landmark. The results of Experiment 2 were clear: When tested without the common landmark and with only two landmarks in Test InNC2, Group Int performed significantly more accurately than Group Con; indeed, the latter group spent no more than a chance amount of time in the platform quadrant.

It is worth noting that several factors might have been expected to militate against the pattern of results observed in these experiments. First, in all cases, Group Int was trained for twice as many trials on the landmark common to the two configurations as on any of the landmarks unique to either. This would presumably have resulted in some overshadowing of the unique landmarks. But since these were the only landmarks present on the critical test trials, this could only have worked to the disadvantage of Group Int. Second, as we already noted in the discussion of Experiment 1, at least one theory of configural learning (that of Pearce, 1994) predicts that there should have been more generalization decrement from training to test trials in Group Int than in Group Con of that experiment. (For Experiment 2, Pearce's model predicts equivalent generalization from training to test configurations in Groups Int and Con.) Finally, there is no reason to believe that it would have been impossible for the animals in Group Con of Experiment 2 to integrate the information provided by their two types of training trials. Although the landmarks and platform were rotated from trial to trial during the course of training, they were rotated to only four different positions, relative to any static background cues that may have been present in the experimental room. Over 96

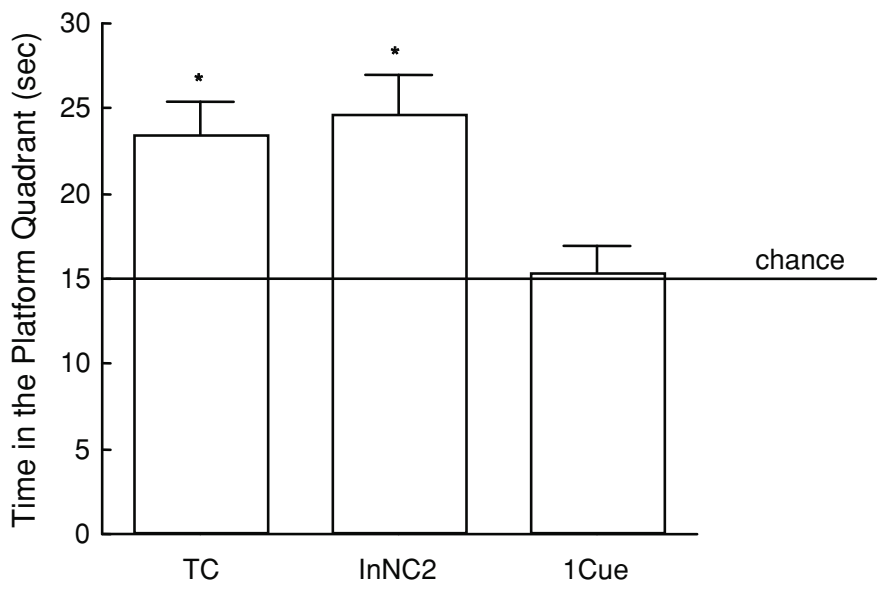

Figure 4. Mean time spent in the platform quadrant by the animals in Experiment 3 during the three tests (TC, InNC2, and 1Cue). 
training trials, it may have been possible for the animals to have learned about each of these four positions, and this would presumably have then allowed them to detect the relationship between Landmarks $\mathrm{A}, \mathrm{B}^{\prime}$, and $\mathrm{C}^{\prime}$ and Landmarks D', E, and D. Moreover, there was, of course, one object that bore a constant relation to the landmarks throughout training - namely, the platform, which was relatively close to Landmarks $\mathrm{D}^{\prime}, \mathrm{E}$, and $\mathrm{D}$ and relatively far away from Landmarks A, B', and $\mathrm{C}^{\prime}$. This should, in principle, have been sufficient for the animals in Group Con to infer the relationship between one set of landmarks and the other. The addition of a landmark common to the two training configurations might be expected to facilitate such an inference, and our results suggest that it did, but it should not have been necessary for any integration to occur.

Our results, therefore, lend support to the conjecture that the map rats construct of any given environment may initially be built up piece by piece, and only at a later stage are the different submaps integrated into a whole map of the entire environment (as proposed by Poucet, 1993). Our evidence suggests that such integration occurs when different submaps share landmarks or features in common that allow their spatial relationship to be inferred. At the same time, it should not be supposed that integration is confined to spatial relationships. Simple conditioning experiments provide good evidence that rats can integrate information from different training episodes, provided that the separate episodes contain some common element. Sensory preconditioning (Brogden, 1939; Razran, 1961) provides one instance of this. Revaluation experiments (Adams \& Dickinson, 1981a, 1981b) provide another.

Perhaps more relevant to the present case is the evidence of sensory preconditioning in the spatial domain provided by Blaisdell and Cook (2005) and by Sawa, Leising, and Blaisdell (2005). In these experiments, pigeons were trained to find, or peck at, an invisible goal, which bore a constant spatial relationship to a particular landmark, LM1. In separate trials, they were exposed to a second landmark, LM2, that bore a constant spatial relationship to LM1. Test trials to LM2 alone revealed that they had been able to infer the spatial relationship between LM2 and the goal. Such findings suggest the following analysis of our results. The training provided for the integration groups in the present experiments with Landmarks A, B, and $\mathrm{C}$ on some trials and $\mathrm{C}, \mathrm{D}$, and $\mathrm{E}$ on others allowed them to learn the spatial relationships between the three landmarks that were presented together on each trial (and of course, their relationship to the hidden platform). But the presence of the common landmark, C, on all the trials also allowed them to infer the spatial relationship between the two sets of unique landmarks - that is, of A and B to D and $\mathrm{E}$. It is this knowledge that allowed them to navigate toward the platform when tested with one unique landmark from each set.

\section{REFERENCES}

AdAms, C. D., \& Dickinson, A. (1981a). Actions and habits: Variations in associative representations during instrumental learning. In N. E. Spear \& R. R. Miller (Eds.), Information processing in animals: Memory mechanisms (pp. 143-165). Hillsdale, NJ: Erlbaum.

Adams, C. D., \& Dickinson, A. (1981b). Instrumental responding following reinforcer devaluation. Quarterly Journal of Experimental Psychology, 33B, 109-121.

Blaisdell, A. P., \& CoOK, R. G. (2005). Integration of spatial maps in pigeons. Animal Cognition, 8, 7-16.

Brogden, W. J. (1939). Sensory pre-conditioning. Journal of Experimental Psychology, 25, 323-332.

Chamizo, V. D., Manteiga, R. D., Rodrigo, T., \& Mackintosh, N. J. (2006). Competition between landmarks in spatial learning: The role of proximity to the goal. Behavioural Processes, 71, 59-65.

Gallistel, C. R. (1990). The organization of learning. Cambridge, MA: MIT Press.

Morris, R. G. M. (1981). Spatial localization does not require the presence of local cues. Learning \& Motivation, 12, 239-260.

Pearce, J. M. (1994). Similarity and discrimination: A selective review and a connectionist model. Psychological Review, 101, 587-607.

PoucET, B. (1993). Spatial cognitive maps in animals: New hypotheses on their structure and neural mechanisms. Psychological Review, 100, $163-182$.

RAZRAN, G. (1961). The observable unconscious and the inferable conscious in current Soviet psychophysiology: Interoceptive conditioning, semantic conditioning, and the orienting reflex. Psychological Review, 68, 81-147.

Sawa, K., Leising, K. J., \& Blaisdell, A. P. (2005). Sensory preconditioning in spatial learning using a touch screen task in pigeons. Journal of Experimental Psychology: Animal Behavior Processes, 31, 368-375.

Spetch, M. L. (1995). Overshadowing in landmark learning: Touchscreen studies with pigeons and humans. Journal of Experimental Psychology: Animal Behavior Processes, 21, 166-181.

Sutherland, R. J., Chew, G. L., Baker, J. C., \& Linggard, R. C. (1987). Some limitations on the use of distal cues in place navigation by rats. Psychobiology, 15, 48-57.

Worden, R. (1992). Navigation by fragment fitting: A theory of hippocampal function. Hippocampus, 2, 165-187.

(Manuscript received April 18, 2006; revision accepted for publication July $31,2006$. 